\title{
Parameters Estimation of the Rakhmatov and Vrudhula Model from the Optimization Method Search in Improved Network
}

\author{
B. F. Silva, P. S. Sausen, and A. Sausen \\ Master's Program in Mathematical Modeling, Regional University of Northwestern Rio Grande do Sul State, 98700000 Ijuí, RS, Brazil \\ Correspondence should be addressed to A. Sausen; airam@unijui.edu.br
}

Received 6 January 2014; Accepted 17 February 2014; Published 23 March 2014

Academic Editors: M.-H. Hsu and K. Jbilou

Copyright ( 2014 B. F. Silva et al. This is an open access article distributed under the Creative Commons Attribution License, which permits unrestricted use, distribution, and reproduction in any medium, provided the original work is properly cited.

\begin{abstract}
This paper presents a proposition of a new optimization method called Search in Improved Network, which is an extension of the method Search in Modified Network, to calculate the empirical parameters of the Rakhmatov and Vrudhula model for predicting batteries lifetime used in mobile devices. The new method is evaluated according to the following methodology. At first empirical parameters are computed considering the optimization methods Search in Improved Network, Search in Modified Network, and Least Squares, as well as the experimental data obtained from a testbed, considering a Lhition-Ion battery, model BL5F, used in the Nokia N95 cell phone. In a second moment, the Rakhmatov and Vrudhula model is validated for each set of parameters obtained, and the simulated data from the model are compared with a set of experimental data. From simulations results a comparative analysis is performed and it is found that by the application of the method Search in Improved Network in the parameters estimation of the Rakhmatov and Vrudhula model it is possible to obtain an easy and intuitive implementation, improving the results obtained in the model accuracy, as well as preserving the runtime.
\end{abstract}

\section{Introduction}

Currently, the use of mobile devices in the most varied areas, such as industry, education, health, and leisure, has been increasing due to the proliferation of wireless technology access. Examples of mobile devices are cell phones, digital cameras, tablets, ipods, laptops, alarm sensors installed in homes or commercial buildings, and other. The market trend is to have smaller mobile devices, faster, which perform the most varied functions, connected to global network of services and resources through a high performance infrastructure.

These devices are powered by a rechargeable battery, so its use is determined through the battery lifetime; it is by definition the time the battery takes to reach the minimum level of power capacity, called cutoff level; after reaching this level the electrochemical reactions cease, and the battery stops providing power to the devices [1].

Thus, in the mobile devices design the battery lifetime is regarded as an important feature, because it reports the time that the device can be used without the need to connect it to an external source. In this context, it is important to have a method to predict the battery lifetime and thus determine the time that the device may remain operating without the need of recharging. One way is by using mathematical models that describe the energy consumption of the devices. Different mathematical models of batteries have been developed in recent years, such as analytical [1-7], stochastic [8], electrical [9-11], and electrochemical models [2], each one with its features and complexity levels. Taking into consideration the related works it is observed that the models with best results for predicting the batteries lifetime are those which consider the nonlinear effects that occur in discharge operations.

Oliveira [4] presents a comparative study among three analytical models used to predict the batteries lifetime: Linear model [12], Peukert's law [12], and Rakhmatov and Vrudhula's diffusion model, called, in this work, RV model [5]. In order to compare these models constant and variable discharge profiles obtained from a testbed are used [13]. In this comparative analysis the models are implemented in the MATLAB computational tool considering parameters of a lithium-ion battery, model BL5F, used in cell phone Nokia N95. From simulations results it is found that the RV model is the most accurate when compared with the other 
simulated models, with average error to predict the battery lifetime of $5.71 \%$ for constant discharges and $6.5 \%$ for variable discharges.

Considering the RV model, it is emphasized that it considers the nonlinear characteristics that occur in a battery, such as the recovery effect and capacity rate effect. In this model the main battery properties are described from Fick's law, where the diffusion process is represented by a partial differential equation (PDE) with boundary conditions of the second kind [5]. It is observed that the RV model has two empirical parameters that depend on the battery used and, therefore, need to be estimated: $\alpha$ which is related to the battery capacity and $\beta$ that is related to nonlinear behavior of the battery.

The estimation of unknown parameters in a mathematical model from experimental data is an inverse problem (IP). According to the literature [14-17] IPs can occur in two different ways: the first is a reconstruction problem; that is, from the effects (measured or observed experimental data) and a mathematical model (i.e., the direct problem (DP)) an unknown cause is searched, and the second is an identification problem; that is, from known causes and effects, the model parameters are sought. In this paper the IP considered is an identification problem.

In the studies that use the RV model for empirical parameters estimation, it is found that all of them use the optimization method Least Squares (LS) batch; that is, the parameters are estimated at once $[1,4-7]$. This method consists in finding the best adjustment for a data set trying to minimize the sum of differences squares between the simulated values by the model and the experimental data obtained from a testbed, in order to maximize the degree of adjustment of the model to the simulated data.

In this context, the aim of this paper is the RV model resolution, for calculating the batteries lifetime, from the proposition of a new optimization method called Search in Improved Network (SIN), which is an extension of the method Search in Modified Network (SMN) proposed by Borges et al. [18] and Cervi [19]. This new method is developed to be applied to the parameters estimation problem of the RV model, aiming at an easier implementation, better accuracy, and good speed of the model.

The remainder of this paper is organized as follows. Section 2 presents the main batteries properties and characteristics, then a state-of-the-art review of mathematical models for predicting the batteries lifetime used in mobile devices is realized, and the concepts of DP and IP, as well as some literature optimization methods, are shown for parameters estimation that are used in this work for comparison with the proposed method. Section 3 presents the proposition of the SIN method for parameters estimation. Section 4 presents the mathematical modeling of DP and IP. Section 5 shows the simulations results and analysis. Finally Section 6 presents the conclusions and suggestions for future work.

\section{State of the Art}

Initially, this section performs a literature review on batteries; it discusses the main types of batteries and their properties and nonlinear characteristics. Then, it presents a literature review of the mathematical models for predicting batteries lifetime used in mobile devices. Finally, the concepts of DP and IP are presented, as well as some literature optimization methods which are used in this paper for comparison with the proposed method.

2.1. Batteries. A battery consists of one or more electrochemical cells that can be connected in series, parallel, or through a combination of both. An electrochemical cell is formed by two electrodes: an anode (negative polarity) and a cathode (positive polarity), separated by an electrolyte. During the discharge period, that is, when the battery provides power to an external circuit, the anode releases electrons for the circuit, while the cathode receives electrons from the circuit. In the battery charging stage, the chemical processes are reversed. The electrons originate from electrochemical reactions and are called electroactive species.

The most popular rechargeable battery technologies for mobile devices include nickel-cadmium (Ni-Cd), nickelmetal hydride (Ni-MH), lithium-ion (Li-Ion), reusable alkaline, and lithium polymer. In this work the battery used is of the type Li-Ion, which is the fastest growing technology today, with energy density significantly higher and a life cycle approximately two times higher compared to Ni-MH batteries. The Li-Ion batteries are more responsive to discharge current characteristics and are often used in laptops, tablets, and cell phones.

Battery has two important properties: (i) voltage, measured in Volts (V), and (ii) capacity, usually measured in Ampere-Hour (Ah); the product of these two variables determines the stored energy amount in the battery. For example, theoretically, a battery of $100 \mathrm{Ah}$ is designed to provide $5 \mathrm{~A}$ for 20 hours or $100 \mathrm{~A}$ during 1 hour. However, this representation is theoretical; in practice, usually the discharge current is not constant in time; that is, discharge operations have nonlinear characteristics, which directly influence the battery lifetime.

The batteries behavior modeling may become complex if nonlinear effects that occur during the discharge period were considered. In the ideal case, the voltage remains constant throughout the discharge period, becoming zero when the battery is discharged. The ideal would be that the capacity to be constant for any discharge current and all stored energy in the battery to be used. On the other hand, in an actual battery discharge procedure, the voltage is slowly reduced and the effective capacity is reduced at high currents. It is emphasized that, depending on the battery type, these effects are of higher or lower impact on its capacity [12].

The batteries have two important nonlinear characteristics. The first one is the recovery effect $[1,5,6,12]$, which is defined by the electrons reorganization in the electrolyte during a relaxation period, that is, time interval in which the discharge current is significantly reduced. In this interval the electrons reorganize themselves in a uniform way, in order that the system recovers the balance and concentration gradient becomes zero in the electrolyte. Thus, the effective capacity of the battery is increased, because a larger charge becomes available before the system reaches the cutoff level. 
The second one is the capacity rate effect that depends on the battery actual capacity and the discharge current intensity; that is, with a high discharge current the battery effective capacity is low, because there is no enough time for the electrons reorganization in the electrolyte (i.e., recovery effect); thus, more charge remains to be used by the system, thereby reducing the capacity and the battery lifetime. For alternating current, the battery effective capacity is increased, because in the change from a high current to a low current, or even for a period with no current, the electrons reorganization occurs in the electrolyte, so that a larger charge on the electrode surface is available, this way, increasing the battery effective capacity.

2.2. Batteries Models. There are different mathematical models that are used in mobile devices designs powered by batteries; they allow for the behavior analysis of charge and discharge under different design specifications. In this section a description is made of the main mathematical models found in the literature that perform this function.

Electrochemical models [2] are based on the chemical processes occurring in the battery and, consequently, are described in detail. This fact makes these models be considered more accurate. However, the detailed description makes them highly complex and difficult to implement due to the large battery parameters amount that must be defined by the user.

Electric models [9-11], also called electrical circuit models, employ a combination of voltage sources, resistors, and capacitors for simulating the battery discharge; they consider the battery capacity rate and its thermal effects. This model presents an easy simulation made from circuit simulators; they are simple to understand and have been widely applied by electrical engineers in analysis of several batteries technologies.

Stochastic models [8] describe the battery in a higher abstraction level than the electrochemical and electrical models. The discharge process is described from stochastic processes. One disadvantage of these models is that they do not consider the nonlinear effects present in a discharge.

Analytical models $[1,4-7,12]$ describe in an abstract way the battery and its main properties, that is, capacity rate effect and recovery effect; they are modeled using a reduced set of equations. This model type has easy implementation when compared to electrochemical and electrical models. The main analytical model found in the literature is RV model; it is described in Section 4 and used in this work for simulating the batteries lifetime.

2.3. Methods of Parameters Estimation. This section presents a brief review of some optimization methods found in the literature, used to estimate the empirical parameters of the RV model. For understanding the methods, initially the concepts of DP and IP are defined, as illustrated in Figure 1.

A DP consists in the precise knowledge of causes for determining the effects, which are the properties computed from a mathematical model, such as temperature, lifetime, electric current, and speed. On the other hand, a IP can be defined in two ways: (i) the first one consists in the determination of unknown causes, from observed or measured

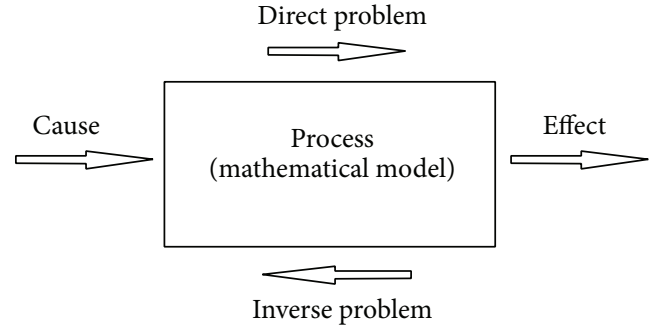

Figure 1: Schematic representation of the PD and PI $[14,20]$.

effects; the causes in a mathematical model are defined as initial conditions, boundary conditions, and source terms; in this case the IP is a reconstruction problem; (ii) the second one consists in determining the systems properties (e.g., parameters), from causes and measured or observed effects; in this case the IP is an identification problem [14-17, 20-22].

A brief description of optimization methods is presented; they are used, in this work, for comparison with the proposed method.

2.3.1. Least Squares Method. Least Squares (LS) method is a procedure to determine the best adjustment to a data set; it realizes minimization of the differences squared sum between the values obtained by the model and the observed data. A general description of the method is presented, with $\zeta$, a vector which contains the parameters $\theta_{n}$ that will be estimated,

$$
\zeta=\left\{\theta_{1}, \ldots, \theta_{n}\right\}^{T},
$$

and a vector $Z$ that contains the inputs $u(N)$ and the outputs $y(N)$ (i.e., experimental measurements) throughout the time interval $1 \leq t \leq N$ given by

$$
Z^{N}=\{u(1), y(1), \ldots, u(N), y(N)\} .
$$

Thus, the LS method consists in the minimizing of an objective function, given by

$$
V_{N}\left(\vec{\zeta}, Z^{N}\right)=\frac{1}{N} \sum_{t=1}^{N}(y(t)-\hat{y}(t \mid \zeta))^{2}
$$

where $y(t)$ is a vector of the measured experimental outputs, $\widehat{y}(t \mid \zeta)$ is a vector of the computed outputs from the model that contains the parameters to be estimated, and $N$ is the experimental data number $[23,24]$.

2.3.2. Method of Search in Modified Network (SMN). The method SMN is proposed by Borges et al. [18] and Cervi [19];it consists in the definition of a range for each parameter to be estimated with a minimum and maximum value that can contain an optimal value of the sought parameter; thus a range network is built. From this network the DP is solved for all the parameters combinations that compose the network; the parameters searched are those that provide the smallest difference between the value calculated by DP and the value experimentally collected. If the optimal parameters values are 
not found, a new range is built from the estimated parameters; the process is redone aiming at a more local search. This method is an extension of the method Search in Network (SN) [16].

The following steps should be followed for the algorithm implementation of the SMN method.

Step 1 . The ranges values $I_{p}=\left[\beta_{p \text { min }}, \beta_{p \max }\right]$ are determined for each parameter $\beta_{p}$, where $p=1,2,3, \ldots, n p$ is the parameters number containing the optimal value $\beta_{p}\left(\beta_{o t}\right)$.

Step 2. A partition of $n c$ data $\beta_{p f}=\beta_{p \min }+(f-1) \Delta \beta_{p}$ is built, with $f=1,2,3, \ldots, n c$ and $\Delta \beta_{p}=\left(\beta_{p \max }-\beta_{p \min }\right) /(n c-1)$.

Step 3. For each value sequence $\left(\beta_{1 f}, \beta_{2 f}, \ldots, \beta_{n p f}\right)$ with $f=$ $1,2,3, \ldots, n c$, the DP is solved.

Step 4. The differences $d_{i}$ are calculated between the estimated solutions and the experimental data from (4) given by

$$
d_{i}=\sum_{j=1}^{z f} \sum_{t=0}^{t f}\left|F_{\mathrm{est}}(j, t)-F_{\exp }(j, t)\right|^{2}
$$

Step 5. The lowest difference $d_{i}\left(d_{\text {min }}\right)$ is identified. This difference corresponds to the parameters set $\beta_{o t}$ for the range $I_{p}$.

Step 6. A new range $I_{p}=\left[\beta_{p \text { min }}, \beta_{p \max }\right]$ is defined for the solution refinement, where $\beta_{p \text { min }}=\beta_{p o t}-\Delta \beta_{p}$ and $\beta_{p \max }=$ $\beta_{\text {pot }}+\Delta \beta_{p}$.

Step 7. Steps 2 and 6 are repeated, so that the ranges are estimated, $I_{p m}, m=1,2,3, \ldots, n r$ (where $n r$ is the refinement number) until

$$
\left|d_{\min }^{i+1}-d_{\min }^{i}\right|<\varepsilon \text { ou } n r=n r_{o t},
$$

where $\varepsilon$ and $n r_{o t}$ are stop criteria.

Following, the optimization method Search in Improved Network (SIN) is presented for the empirical parameters estimation $\alpha$ and $\beta$ of the RV model from experimental data obtained from a testbed.

\section{Method of Search in Improved Network (SIN)}

Throughout the study and the implementation of the SMN method, it is found that it has a limitation; because it is a suboptimal search method the sought parameter must necessarily be within the predefined range. In case that no prior knowledge of this value is present, the range must be large enough to contain the sought parameter. In these cases the search for the parameter may become a rather time consuming process.

In this context, the SIN method is made from a modification in the SMN method that aims to eliminate the condition that the parameter needs to be within the range. In the new method the ranges are created from an initial value for each estimated parameter, after a network of ranges is built, so that more accurate values for parameters are sought. In case that the parameters are not contained in the initially determined ranges, this process is redone from the best parameter found in the previous iteration that will be caught as a new initial value. It is observed that at the beginning of the simulation a convergence criterion is established, so that from any initial value there will be an orientation to the networks to find the optimal value for each parameter. Following, the description of the method is presented.

\subsection{Method Description}

Step 1. Initial values $\theta_{1}, \theta_{2}, \ldots, \theta_{n}$, for each estimated parameter, are determined, where $n$ is the parameters number.

Step 2. A range

$$
\operatorname{Int}_{n}=\left[\theta_{n \text { inf }}, \ldots, \theta_{n \text { sup }}\right]
$$

for each parameter is built from initial values $\theta_{n}$ and of the choice of a unit rate for $\rho$ that will determine the amplitude of the ranges. The lower limit of the range $\mathrm{Int}_{n}$ is given by

$$
\theta_{n \text { inf }}=\theta_{n}-\rho \theta_{n}
$$

and the upper limit of the range is given by

$$
\theta_{n \text { sup }}=\theta_{n}+\rho \theta_{n}
$$

Step 3. A partition of points in the ranges $\mathrm{Int}_{n}$ is built, where the amount of points $q$ defines the size of the spacing $\lambda$ that is given by

$$
\lambda=\frac{\left(\theta_{n \text { sup }}-\theta_{n \mathrm{inf}}\right)}{q-1},
$$

so each range is written in the following form:

$$
\begin{aligned}
\text { Int }_{n}=[ & \theta_{n \text { inf }}, \theta_{n \text { inf }}+\lambda, \theta_{n \text { inf }}+2 \lambda, \ldots, \theta_{n \text { inf }} \\
& \left.+(q-1) \lambda, \theta_{n \text { sup }}\right] .
\end{aligned}
$$

Step 4. The DP is solved considering all parameters combinations contained in the ranges.

Step 5. The differences $d_{i}$ between the estimated solutions $S_{\text {est }}$ and the experimental data $S_{\text {exp }}$ are calculated for each parameters combination using the equation

$$
d_{i}=\sum_{i=1}^{N}\left|S_{\exp _{i}}-S_{\text {est }}\right|^{2}
$$

where $N$ is the experimental data number.

Step 6. The lowest value of $d_{i}\left(d_{\text {min }}\right)$ is identified. This value corresponds to the parameters set $\theta_{o t}$ which is located in ranges Int $_{n}$ built from the proposed initial values $\theta_{1}, \theta_{2}, \ldots, \theta_{n}$.

Step 7. In possession of $\theta_{n, o t}$ it is verified whether the difference between the experimental data and the calculated values 
by the model is less than a previously established error $(\varepsilon)$; if so, the parameters values are found.

Otherwise, the stop criteria are used:

$$
\left|d_{\min }^{i+1}-d_{\min }^{i}\right|<\xi \text { ou } n r=n i_{o t},
$$

where $\xi$ and $n i_{o t}$ (iterations number) are determined by the user and Steps 2 to 6 are repeated until the error is satisfied.

Next section presents the DP mathematical modeling, that is, the RV model, and the IP.

\section{Mathematical Modeling}

4.1. Direct Problem. This section presents the RV model which considers the nonlinear effects that occur in batteries. Concentration behavior due to one-dimensional diffusion is described by the following Fick's laws:

$$
\begin{aligned}
-J(x, t) & =D \frac{\partial C}{\partial x}(x, t), \\
\frac{\partial C}{\partial t}(x, t) & =D \frac{\partial^{2} C}{\partial x^{2}}(x, t),
\end{aligned}
$$

where $J(x, t)$ denotes the species flux at time $t$ at distance $x$ of the electrode and $D$ denotes the diffusion coefficient. Let $C(x, t)$ be the electroactive species concentration at time $t \in[0, L]$ and at distance $x \in[0, w]$ of the electrode. It is considered a battery with full charge (i.e., $t=0$ ); the species concentration is constant through the electrolyte length, so the initial condition is given by

$$
C(x, 0)=C^{*} \text {. }
$$

The battery is considered discharged when $C(0, t)$ is lower than cutoff level. In accordance with Faraday's law, the flux at the boundary of the diffusion region $(x=0)$ is proportional to the current $i(t)$ (i.e., the external charge applied), and flux in the other boundary of the diffusion region $(x=w)$ is zero. These propositions provide the following boundary conditions:

$$
\begin{gathered}
\left.D \frac{\partial C}{\partial x}(x, t)\right|_{x=0}=\frac{i(t)}{v F A}, \\
\left.D \frac{\partial C}{\partial x}(x, t)\right|_{x=w}=0
\end{gathered}
$$

where $A$ denotes the surface area of the electrode, $F$ denotes Faraday's constant, and $v$ denotes the electrons number involved in the electrochemical reaction at the electrode surface.

Oliveira [4] performs the equation resolution (14) with initial condition given by (15) and boundary conditions given in (16) through the use of Laplace transform and Laplace inverse transform for obtaining the equation

$$
\alpha=\int_{0}^{L} \frac{i(\tau)}{\sqrt{L-\tau}} d \tau+2 \sum_{n=1}^{\infty} \int_{0}^{L} \frac{i(\tau)}{\sqrt{L-\tau}} e^{-\beta^{2} n^{2} /(L-\tau)} d \tau .
$$

From (17) it is observed that RV model may be used to calculate the batteries lifetime using constant or variable discharges. In this context, a constant discharge current $i(t)=$ $I$ is considered, so (17) turns into

$$
\begin{aligned}
I=\alpha(2 \sqrt{L} & \\
\times & \times 1+2 \sum_{n=1}^{\infty}\left(e^{-\beta^{2} n^{2} / L}\right. \\
& \quad\left(\pi e^{-\beta^{2} n^{2} / L}\right. \\
& \times(\pi-1 \\
& \left.\left.\left.\left.\left.+\sqrt{1+\frac{\pi L}{\beta^{2} n^{2}}}\right)^{-1}\right)\right)\right]\right)^{-1} .
\end{aligned}
$$

The easy implementation requiring both experimental data of a real problem and an inverse routine for empirical parameter estimation is one of the main features of the RV model. The RV model equation (18) relates the discharge current $I$ with the battery lifetime $L$ and the empirical parameters $\alpha$ and $\beta$ which need to be estimated, because they depend on the battery used.

4.2. Inverse Problem. The IPs analysis is a multidisciplinary research area, with a large number of applications in different fields of science. Different researches about this issue may be found; they show that the error analysis together with an inverse routine may be satisfactory for the parameters estimation [14-17, 20, 24].

In this paper the IP considered is formulated as an optimization problem, which seeks to minimize the objective function of the squared residuals given by

$$
Q(\vec{Z})=\sum_{i=1}^{N}\left(I_{\text {est }_{i}}(\vec{Z})-I_{\exp _{i}}\right)^{2}=\left(\vec{E}_{i}\right)^{T} \vec{E}_{i}
$$

where $\vec{E}_{i}=I_{\text {est }_{i}}(\vec{Z})-I_{\exp _{i}}, I_{\text {est }_{i}}(\vec{Z})$ denotes the discharge current calculated by the model and $I_{\text {est }_{i}}$ denotes the experimental discharge current, $N$ is the measured experimental data number, and $\vec{Z}$ denotes a vector that contains the estimated parameters, given by

$$
\vec{Z}=\{\alpha, \beta\}^{T}
$$

The next section presents the resolution of the IP considering the SIN, SMN, and LS methods, as well as the simulations results, comparison, and analysis.

\section{Simulations Results and Analysis}

This section presents the simulations results of the SIN optimization method applied to the RV model, for calculating 


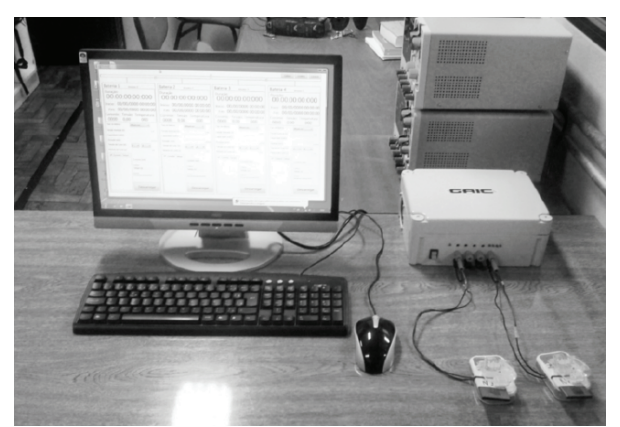

FIgURE 2: Testbed.
TABLE 1: Data used for the parameter estimation of the RV model [6].

\begin{tabular}{lc}
\hline$I(\mathrm{~mA})$ & $L_{m}(\mathrm{~min})$ \\
\hline 100 & 483.71 \\
150 & 325.67 \\
250 & 192.32 \\
350 & 136.25 \\
450 & 105.7 \\
550 & 85.8 \\
650 & 70.73 \\
750 & 60.74 \\
850 & 51.7 \\
950 & 46.7 \\
\hline
\end{tabular}

TABLE 2: Data used for the RV model validation [4].

the empirical parameters $\alpha$ and $\beta$, from the MATLAB computational tool. Then, it presents the simulations results from using the MQ and SMN optimization methods also applied to the RV model for the computing of the empirical parameters $\alpha$ and $\beta$. Finally, a comparative study between the results found is realized to validate the proposed method.

A set of actual data, which consists in the battery discharging process, is needed to perform the parameters estimation of the RV model. Figure 2 presents the testbed that provides a complete environment for the realization of physical experiments involving batteries. Further details of this experiment can be found in [13]. The batteries used are of the Li-Ion technology, model BL5F, used in the cell phone Nokia N95.

The same standard for performing experimental tests is adopted, in order to minimize any differences in the end result of the tests. First, the discharge profiles are defined following the same procedure used by Rakhmatov and Vrudhula [5]. Thus, in this work the profiles used are composed of ten constant discharge currents that are measured in milliamperes $(\mathrm{mA})$. The values of the currents are distributed uniformly in relation to the battery full charge capacity, including low, medium, and high charges.

The methodology used to collect data from the testbed is described below. The starting point is the fully charged battery (i.e., voltage from 4.2 volts). The experiments are divided into two moments: the first one is done with ten constant discharge currents; the results are presented in Table 1; they are used for the parameters estimation of the RV model; the second one is done with three constant discharge currents; the results are shown in Table 2; they are used for RV model validation. The discharge profile $(I)$ is presented in the first column and the battery average lifetime $\left(L_{M}\right)$ is presented in the second column, for both tables. For each discharge profile the tests are repeated ten times for obtaining of a satisfactory statistical sampling.

The parameters $\alpha$ and $\beta$ are estimated initially from the SIN method that seeks a set of parameters for minimizing (19), from the DP shown in (18). According to [5], (18) of the DP is very complex to solve by isolating $L$; then it is assumed that $L$ is obtained from testbed and it is tried to predict the value of $I$ instead. The parameter $n$, in the summation of (18), represents the experimental data number from the testbed. In

\begin{tabular}{lc}
\hline$I(\mathrm{~mA})$ & $L_{m}(\mathrm{~min})$ \\
\hline 200 & 268.13 \\
500 & 268.13 \\
700 & 62.68 \\
\hline
\end{tabular}

this work 10 experimental datasets are considered; this value is suggested by Rakhmatov and Vrudhula in [5].

Table 3 shows the iterations of SIN method algorithm. The methodology requires that, initially, an initial value is allocated for each parameter; in this paper $\alpha=1$ and $\beta=1$ are chosen, as it is shown in iteration 0 . The range size and the spacing among points are defined by the user; a limit of $\pm 300 \%$ is applied, so that $\rho=3$ and $q=100$, to define the spacing. It is emphasized that the ranges presented are not the only inspected ones. During the method execution 45 ranges are inspected, but only 9 of them are significant, since they contributed to the algorithm evolution by finding a parameters set with less error than the previous one. The runtime of the method is approximately 10 seconds. Table 4 shows the values for $\alpha$ and $\beta$ from SIN method.

After the parameters calculation of $\alpha$ and $\beta$ from the SIN optimization method, the battery lifetime is calculated by RV model in (18) and from the constant discharge currents shown in Table 2. Next, the lifetime simulated by the model $\left(L_{s}\right)$ is compared with the average experimental lifetime $\left(L_{m}\right)$ presented in Table 2. Table 5 shows the results comparison.

The model validation shows that at lower charges (e.g., $200 \mathrm{~mA}$ ) the RV model presented lower accuracy when compared to high charges of $700 \mathrm{~mA}$. This fact occurs because at discharge currents of low intensity there is a longer battery discharging time; thereby the process is submitted to the system nonlinearities. In the simulations results of the RV model an average error of $5.27 \%$ is obtained.

Table 6 presents the $\alpha$ and $\beta$ parameter values from the LS method. It is observed that the running time of this method is instantaneous $[1,4-6]$.

After the parameters calculation of $\alpha$ and $\beta$ from the LS optimization method, the battery lifetime is calculated by RV model in (18) and from constant discharge currents shown in Table 2. Next, the lifetime simulated by the model 
TABLE 3: Results from SIN method execution.

\begin{tabular}{|c|c|c|c|c|c|c|}
\hline Iteration & $\operatorname{Lim}_{\text {inf }} \alpha$ & $\alpha_{o t}$ & $\operatorname{Lim}_{\text {sup }} \alpha$ & $\operatorname{Lim}_{\text {inf }} \beta$ & $\beta_{o t}$ & $\operatorname{Lim}_{\text {sup }} \beta$ \\
\hline 0 & & 1 & & & 1 & \\
\hline 1 & -2 & 4 & 4 & -2 & 4 & 4 \\
\hline 2 & -8 & 16 & 16 & -8 & 16 & 16 \\
\hline 3 & -32 & 64 & 64 & -32 & 64 & 64 \\
\hline 4 & -128 & 256 & 256 & -128 & 256 & 256 \\
\hline 5 & -512 & 1024 & 1024 & -512 & 256 & 1024 \\
\hline 6 & -2048 & 4096 & 4096 & -512 & 256 & 1024 \\
\hline 7 & -8192.00 & 10674.42 & 16384.00 & -512.00 & 15.52 & 1024.00 \\
\hline 8 & -21348.85 & 19408.04 & 42697.70 & -31.03 & 4.70 & 62.06 \\
\hline 9 & -38816.09 & 18819.92 & 77632.18 & -9.40 & 4.84 & 18.81 \\
\hline
\end{tabular}

TABLE 4: RV model parameters from the SIN method.

\begin{tabular}{|c|c|c|}
\hline Method & \multicolumn{2}{|c|}{ SIN } \\
\hline Parameter & $\alpha$ & $\beta$ \\
\hline Value & 18820 & 4.84 \\
\hline
\end{tabular}

TABLE 5: RV model validation from parameters calculated by SIN method.

\begin{tabular}{lcccc}
\hline$I(\mathrm{~mA})$ & $L_{m}(\mathrm{~min})$ & $L_{s}(\mathrm{~min})$ & Error & Error $(\%)$ \\
\hline 200 & 268.13 & 247.22 & 20.90 & 7.79 \\
500 & 98.23 & 94.33 & 3.89 & 3.96 \\
700 & 62.68 & 65.22 & 2.54 & 4.05 \\
& Average error (\%) & & $\mathbf{5 . 2 7}$ \\
\hline
\end{tabular}

TABLE 6: RV model parameters from the LS method.

\begin{tabular}{lccc}
\hline Method & \multicolumn{2}{c}{ LS } \\
Parameter & $\alpha$ & & $\beta$ \\
Value & 19993 & & 4.5 \\
\hline
\end{tabular}

TABLE 7: RV model validation from parameters calculated by LS method.

\begin{tabular}{lcccc}
\hline$I(\mathrm{~mA})$ & $L_{m}(\min )$ & $L_{s}(\mathrm{~min})$ & Error & Error (\%) \\
\hline 200 & 268.13 & 245 & 23.13 & 8.62 \\
500 & 98.23 & 94 & 4.23 & 4.30 \\
700 & 62.68 & 65.33 & 2.65 & 4.23 \\
& Average error $(\%)$ & & $\mathbf{5 . 7 2}$ \\
\hline
\end{tabular}

$\left(L_{s}\right)$ is compared with the average experimental lifetime $\left(L_{m}\right)$ presented in Table 2. Table 7 presents the results comparison.

Similarly to the previous simulations results, the RV model validation shows that at lower charges (e.g., $200 \mathrm{~mA}$ ) the RV model presented lower accuracy when compared to high charges of $700 \mathrm{~mA}$. This fact occurs because at discharge currents of low intensity there is longer battery discharging time; thereby the process is submitted to the system nonlinearities. In simulations results of the RV model an average error of $5.72 \%$ is obtained.

Finally, the parameters $\alpha$ and $\beta$ are estimated from the SMN optimization method that seeks a set of parameters for minimizing (19), from the DP shown in (18). Table 1 presents data used for parameter estimation. The execution procedure of this method requires that, initially, ranges to be defined may contain the optimal values of the estimated parameters. This task to find the valid ranges is a method empirical characteristic; in case that the user does not know the process, the search can reach no satisfactory result. Thus, it is suggested that the initial search is to be made in a range with large dimensions and then get closer to the optimal value with ever shorter ranges.

Table 8 presents the parameter estimation results by the SMN method, in which the selected intervals in each search are specified, as well as the best value found for each parameter in interval. It is observed that after the tenth execution the algorithm converged to the values of $\alpha$ and $\beta$ sought. In total, 13 algorithm executions are carried out. It is emphasized that the algorithm could continue to run, seeking a better value for the parameter, but this does not ensure finding a value with the lowest error required.

The algorithm methodology requires the user to define a divisions number for the proposed ranges. Thus, during the tests for the algorithm implementation the value of 100 divisions demonstrated to have the best result of time versus accuracy. The SMN method lost precision for a value below 100. In contrast, a value above 100 resulted in an algorithm runtime increase, without increasing significantly the accuracy. The total execution time of the SMN method, by considering the required analysis, is approximately 20 hours. Table 9 shows the values of $\alpha$ and $\beta$ from the SMN method.

After the parameters calculation of $\alpha$ and $\beta$ from the SMN optimization method, the battery lifetime is calculated by RV model in (18) and from constant discharging currents shown in Table 2. Following, the simulated lifetime by the model $\left(L_{s}\right)$ is compared with the average experimental lifetime $\left(L_{m}\right)$ presented in Table 2. Table 10 shows the results comparison.

Similarly to the previous simulations results, looking at the obtained errors, the RV model validation shows that at lower charges (e.g., $200 \mathrm{~mA}$ ) the RV model presented lower accuracy when compared to high charges of $700 \mathrm{~mA}$. This fact occurs because at discharging currents of low intensity there is a longer battery discharging time; thereby the process is submitted to the system nonlinearities. In the simulations results of the RV model an average error of $6.53 \%$ is obtained. 
TABLE 8: Results from SMN method execution.

\begin{tabular}{|c|c|c|c|c|c|c|c|}
\hline Iteration & $\operatorname{Lim}_{\text {inf }} \alpha$ & $\alpha_{o t}$ & $\operatorname{Lim}_{\text {sup }} \alpha$ & $\operatorname{Lim}_{\text {inf }} \beta$ & $\beta_{o t}$ & $\operatorname{Lim}_{\text {sup }} \beta$ & Tempo \\
\hline 1 & -100000 & 10800 & 100000 & -100000 & 10800 & 100000 & 600 \\
\hline 2 & 0 & 10830 & 30000 & 0 & 10830 & 30000 & 59 \\
\hline 3 & 0 & 9990 & 10000 & 0 & 29970 & 30000 & 62 \\
\hline 4 & 0 & 9990 & 10000 & 0 & 999 & 1000 & 68 \\
\hline 5 & 0 & 9990 & 10000 & 0 & 99.9 & 100 & 170 \\
\hline 6 & 0 & 19980 & 20000 & 0 & 99.9 & 100 & 92 \\
\hline 7 & 0 & 19920 & 30000 & 0 & 6.64 & 10 & 68 \\
\hline 8 & 10000 & 20000 & 30000 & 3 & 6.5 & 10 & 69 \\
\hline 9 & 15000 & 19650 & 30000 & 0 & 3.1 & 10 & 1.2 \\
\hline 10 & 15000 & 20000 & 25000 & 0 & 5 & 10 & 1.2 \\
\hline 11 & 18000 & 19920 & 22000 & 3 & 4.92 & 7 & 1.2 \\
\hline 12 & 19000 & 19920 & 21000 & 4 & 4.92 & 6 & 1.2 \\
\hline 13 & 19500 & 19990 & 20500 & 4.5 & 4.99 & 5.5 & 1.2 \\
\hline
\end{tabular}

TABLE 9: RV model parameters from SMN method.

\begin{tabular}{|c|c|c|}
\hline Method & \multicolumn{2}{|c|}{ SMN } \\
\hline Parameter & $\alpha$ & $\beta$ \\
\hline Value & 19990 & 4.99 \\
\hline
\end{tabular}

TABLE 10: RV model validation from parameters calculated by SMN method.

\begin{tabular}{lcccc}
\hline$I(\mathrm{~mA})$ & $L_{m}(\mathrm{~min})$ & $L_{s}(\mathrm{~min})$ & Error & Error $(\%)$ \\
\hline 200 & 268.13 & 317.92 & 49.79 & 18.57 \\
500 & 98.23 & 112.23 & 14 & 14.26 \\
700 & 62.68 & 66.69 & 4.01 & 6.4 \\
& Average error $(\%)$ & & $\mathbf{6 . 5 3}$ \\
\hline
\end{tabular}

The simulation results show that the SIN method has an accessible implementation and good runtime speed, compared to other simulated methods. The accessibility in relation to its implementation consists in the operation, which from 10 experimental discharges profiles of the battery and of the objective function, the parameters estimation of the RV model is performed. The SIN method algorithm performs the setting of the networks from an initial value for the parameters, and it searches in these networks for the best value. In case that the value found does not satisfy the error required, the algorithm builds a new network and performs a new search from the obtained values for the parameters in the previous iteration. This process is done in an exhaustive way enabling the method to converge to best parameter value.

By performing a comparison between the simulations results of the SIN method and the LS method for the RV model validation, it is observed that both methods have a very small difference in relation to accuracy; that is, the first one presents an average error of 5.27\%, and the second one presents an average error of $5.72 \%$. It is highlighted that the implementation of the SIN method is more simple than the LS method, because it uses an algorithm of search from the executed objective function in an exhaustive way, by requiring only input and output data obtained through experiments performed at a testbed.

A comparison between the simulations results of the SIN method and the SMN method for the RV model validation is performed, so a percentage difference between the errors is verified. The SIN method has greater accuracy with an average error of $5.27 \%$ when compared to the SMN method, with average error of $6.53 \%$. This difference occurs due to different search methodology for the parameters. The SMN method performs searches on suggested networks from observations of the discharging profiles and afterwards it carries out a reduction in the width of the ranges based on the user decisions, whereas the SIN method performs searches on suggested networks from an initial value for the parameters and afterwards the algorithm moves itself without the user's interference. In this work the SIN method carried out 40 searches, even so the runtime is not affected; while the SIN method took 10 seconds to run, the SMN method took around 20 hours.

\section{Conclusions}

This paper presented a new optimization method proposition for the parameters estimation of the RV model, called Search in Improved Network. The main feature of the method is to perform parameters estimation using both the experimental data and the objective function of the model. It is noted that the parameters are computed from simple and dynamic way.

The new method was evaluated following the methodology: first, the parameters $\alpha$ and $\beta$ of the RV model were estimated by considering the optimization methods SIN, LS, and SMN, as well as the experimental data obtained from the testbed, for Li-Ion batteries, model BL5F, used in Nokia 95 cell phone; second, the RV model was validated for each parameters set, and the simulated data from the RV model were compared with the experimental data, and finally a comparative analysis of the obtained results was performed.

From the comparative analysis between the simulations results of the RV model, considering the optimization methods SIN, LS, and SMN, it is concluded that the SIN 
method showed the best result for the model accuracy, that is, average error of 5.27. It has easy implementation and good execution speed. Therefore, it can be recommended for parameters estimation in other areas of science.

\section{Conflict of Interests}

The authors declare that there is no conflict of interests regarding the publication of this paper.

\section{Acknowledgment}

The authors acknowledge the financial support to the Agency Fapergs from Edict 02/2011 (PQG).

\section{References}

[1] P. Sausen, Energy integrated management and topology control in wireless sensor networks [Ph.D. thesis], UFCG, Campina Grande, Brazil, 2008.

[2] K. Lahiri, A. Raghunathan, S. Dey, and D. Panigrahi, "Batterydriven system design: a new frontier in low power design," in Proceedings of the 7th Asia and South Pacific and the 15th International Conference on VLSI Design (ASP-DAC '02), pp. 261-267, 2002.

[3] T. L. Martin, Balancing batteries, power, and performance: system issues in CPU speed-setting for mobile computing [M.S. thesis], Carnegie Mellon Unerversity, 1999.

[4] A. V. Oliveira, Comparative analysis of methodologies of parameter estimation applied in analytical models used in the prediction of the battery lifetime [M.S. thesis], Northwest Regional University of Rio Grande do Sul State, Ijuí, Brazil, 2012, (Portuguese).

[5] D. N. Rakhmatov and S. B. K. Vrudhula, "An analytical high-level battery model for use in energy management of portable electronic systems," in Proceedings of the IEEE/ACM International Conference on Computer-Aided Design, pp. 488493, November 2001.

[6] K. K. Schneider, Analytical models for predicting of battery lifetime used in mobile devices [M.S. thesis], Northwest Regional University of Rio Grande do Sul State, Ijuí, Brazil, 2011, (Portuguese).

[7] K. K. Schneider, P. S. Sausen, and A. Sausen, "Comparative analysis of the batteries lifetime used in mobile devices from the analytical models," TEMA, Trends in Applied and Computational Mathematics, vol. 12, no. 1, pp. 43-54, 2011.

[8] C. Chiasserini and R. Rao, "A model for battery pulsed discharge with recovery effect," in Proceedings of the Wireless Communications and Networking Conference, pp. 636-639, 1999.

[9] M. Chen and G. A. Rincón-Mora, "Accurate electrical battery model capable of predicting runtime and I-V performance," IEEE Transactions on Energy Conversion, vol. 21, no. 2, pp. 504511, 2006.

[10] C. M. D. Porciuncula, Application of battery electric models for predicting of the lifetime of mobile devices [M.S. thesis], Northwest Regional University of Rio Grande do Sul State, Ijuí, Brazil, 2012, (Portuguese).

[11] C. M. D. Porciuncula, A. Oliveira, P. S. Sausen, and A. Sausen, "Comparative evaluation between the battery electric model, and linear and peukert law analytical models," Journal Brazilian of Applied Computing, vol. 4, no. 1, pp. 71-80, 2012 (Portuguese).
[12] M. Jongerden and B. Haverkort, "Battery modeling," Tech. Rep., Faculty Electrical Engineering, Mathematics and Computer Science, January 2008.

[13] H. B. Nonemacher, L. Minelli, P. S. Sausen, and A. Sausen, "Development of a test bed for evaluating of mathematical models to predict the batteries lifetime," in XXIV CRICTE, Regional Conference of Undergraduate Research and Technological Engineering, Rio Grande, RS, Brazil, 2010, (Portuguese).

[14] A. de Cezaro, Inverse Problems: An Introduction, Mathematics Colloquium in the South Region, 1, SBM, 2010, (Portuguese).

[15] M. I. Mesa, O. L. Santiago, F. H. Fernández Accurate, D. C. Rodríguez, A. J. da Silva Neto, and L. D. T. Câmara, "An approach to parameters estimation of a chromatography model using a clustering genetic algorithm based inverse model," Soft Computing, vol. 15, no. 5, pp. 963-973, 2011.

[16] A. J. Silva Neto, Inverse Problems: Fundamental Concepts and Applications, UERJ, Rio de Janeiro, Brazil, 2005, (Portuguese).

[17] A. Tarantola, Inverse Problem Theory and Methods for Model Parameter Estimation, SIAM, Paris, France, 2005.

[18] P. A. P. Borges, M. T. Vione, and A. Cervi, "Determination of coefficients of the van Genuchten model by employing inverse problem," in Proceedings of VIII Regional Meeting of Applied Mathematics and Computational, Pelotas, 2008, (Portuguese).

[19] A. Cervi, Determination of the characteristic equation parameters of soils through the inverse problems solution technical based on data evaporation [M.S. thesis], Northwest Regional University of Rio Grande do Sul State, Ijuí, Brazil, 2009, (Portuguese).

[20] A. J. Silva Neto and J. C. Becceneri, Intelligence Computational Techniques Inspired in the Nature: Applications in Inverse Problems in Radiative Transfer, Notes in Applied Mathematics, 41, 2009, (Portuguese).

[21] H. W. Engl, M. Hanke, and A. Neubauer, Regularization of Inverse Problems: Mathematics and Its Applications, Mathematics and Its Applications Series, Kluwer Academic Plubishers, 1996.

[22] N. M. McCormi, "Inverse problems: methods and applications," TEMA, Trends in Applied and Computational Mathematics, vol. 13, no. 3, pp. 277-289, 2012.

[23] L. A. Aguirre, Introduction to System Identification: Linear and nonLinear Techniques Applied to Real Systems, UFMG, Belo Horizonte, Brazil, 2007, (Portuguese).

[24] L. Ljung, System Identification Theory for the User, Prentice Hall, Englewood Cliffs, NJ, USA, 1999. 


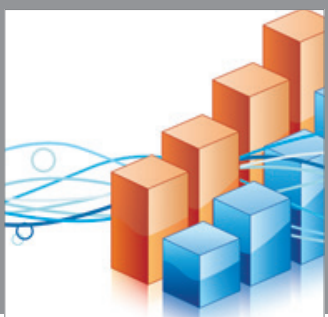

Advances in

Operations Research

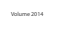

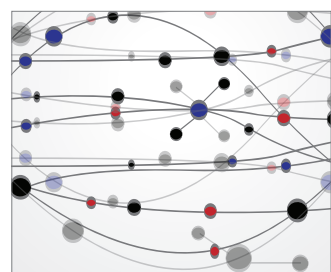

The Scientific World Journal
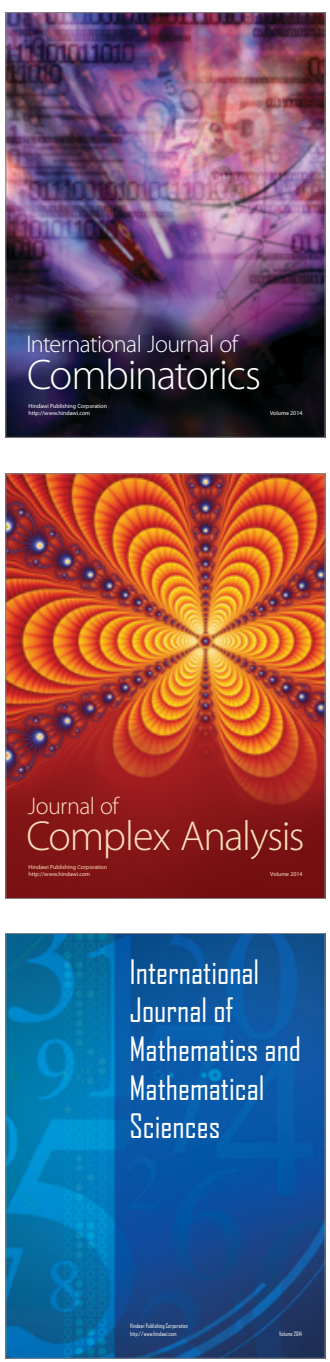

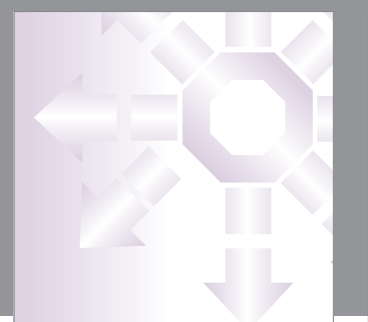

Advances in

Decision Sciences

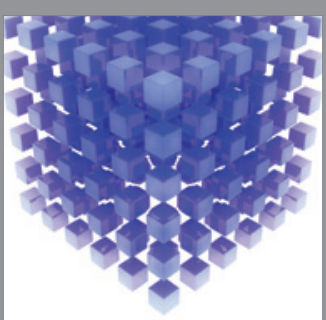

Mathematical Problems in Engineering

vament

\section{Algebra}

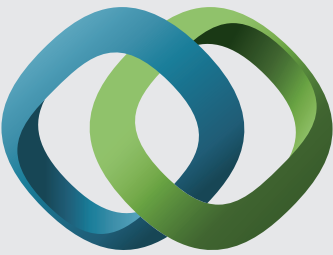

\section{Hindawi}

Submit your manuscripts at http://www.hindawi.com
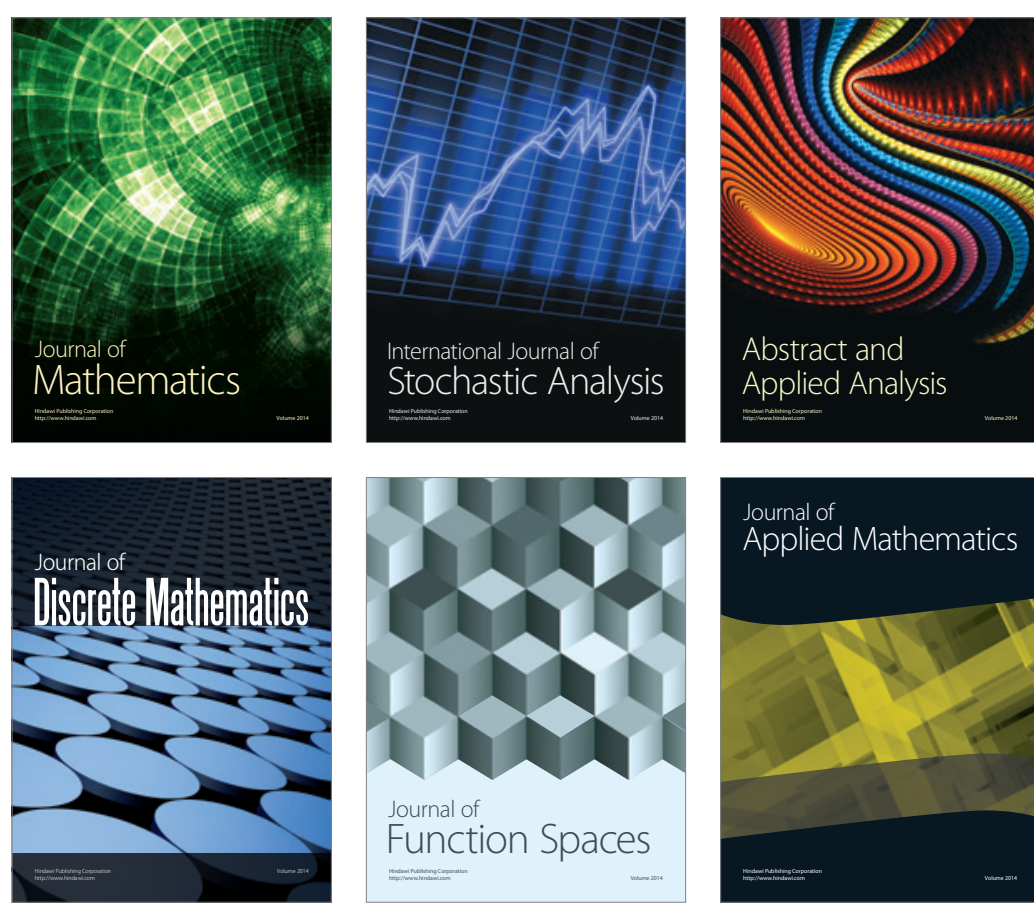

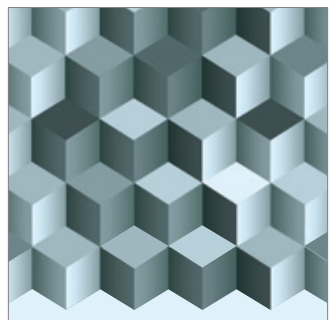

Journal of

Function Spaces

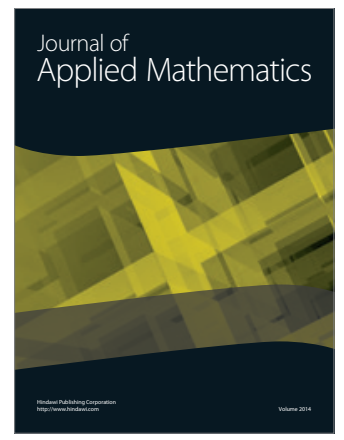

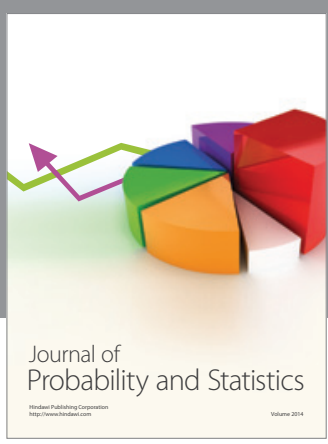
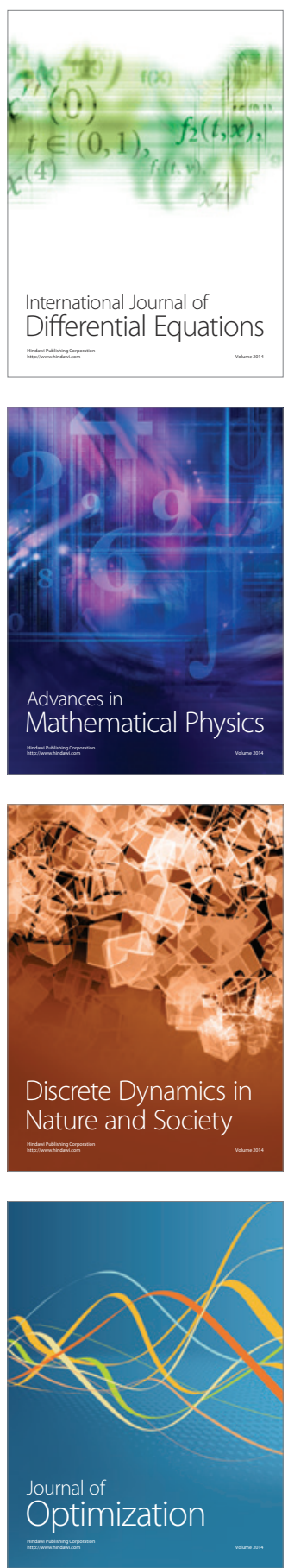